\title{
BENEFIT REALISATION MODELLING FOR ERP SYSTEMS USING SYSTEM
} DYNAMICS

\author{
Amgad Badewi \\ Manufacturing and Materials Department \\ Cranfield University, Cranfield \\ Bedford, MK43 0AL, UK \\ a.badewi@cranfield.ac.uk
}

\author{
Essam Shehab \\ Manufacturing and Materials Department \\ Cranfield University, Cranfield \\ Bedford, MK43 0AL, UK \\ e.shehab@cranfield.ac.uk
}

\author{
Joe Peppard \\ School of Management \\ Cranfield University, Cranfield \\ Bedford, MK43 0AL \\ j.peppard@cranfield.ac.uk
}

\begin{abstract}
Achieving success for ERP systems is not only concerned with deploying the technology on time and on budget but also concerned with the level of achieving targeted benefits from the system. This level of achieving targeted benefits can be managed indirectly through managing the organisational attitude toward the new system. Thus, the aim of this paper is to use the System Dynamics (SD) to reveal how managing organisational attitude leads to an increase in the level of system use and, therefore, leads to a decrease in the level of resistance to change to new system for achieving high level of performance. SD is used to investigate the causal loops of organisational attitude, perceptions, behaviours, and policies taken to achieve desired benefits. The ability to manage an organisational attitude toward ERP is an important factor for achieving desired levels of benefits is the main conclusion of this research.
\end{abstract}

Keywords: ERP, System Dynamics, Benefit Realisation.

\section{INTRODUCTION}

Enterprise Resource Planning (ERP) is an enterprise wide system that integrates information across various departments. Like the new management system, ERP system could face resistance by the stakeholders such as employees, customers, and vendors. This resistance could be because of the fear of changes that could affect their job nature, fear of the inability to use the system easily, and fear that this new change will not add value to their work or even harm the work. These attitudes, and also their consequence behaviour of resistance, come from the perception of, or attitude toward, this new technology. As a result, the ability to manage these attitudes through managing perceptions could improve the adaptability process of new ERP system.

About seventy per cent of new Information Systems (IS) projects could not achieve the targeted performance (Clegg et al., 1997). One of the reasons that explain this phenomenon is the care after implementing these systems. Most of ERP vendors, and the adopting organisation, consider their ERP implementation projects finished just after delivering the system and training employees. In fact, after ERP implementation, the performance of organisation declines shortly because of the adaptation process (Shang and Seddon, 2000). The performance needs on average from 2 to 3 years in order to increase the performance significantly (Nicolaou, 2004). Nevertheless, this is not the case for all ERP adopting organisations; many of them fail and go to bankruptcy after implementing ERP (Davenport, 1998). This is an indication that organisations that adopt ERP should manage the post-implementation period effectively in order to manage this risk as well as being able to manage the decline in the 
performance in a good manner. There are factors important for the effectiveness of benefit management (Breese, 2012) such as organisational culture, timing and capacity for change, and governance and ownership. Organisational culture, timing and capacity for change, and governance and ownership are three factors that are covered in this research. Ownership problem which focuses on who is the responsible for achieving, tracking, and measuring benefits? Therefore, it is addressed that benefit Realisation process should be done in project (Breese, 2012; Pellegrinelli et al., 2011; Bradley, 2010) called in this research Benefit Realisation Project (BRP) team.

Since ERP benefit Realisation project, as any organisational change, could have an impact on the organisation performance, the results should be simulated first before the project starts. Not only simulation matters, but also the indicators used and the required actions by BRP team should be addressed.

A system dynamics model looks like a control system that has thermostat in order to manage a certain process (Coyle, 1996). The model consists of indicators, actions, and intermediates. Intermediates are mainly the perceptions of the user. BRP team has controllable variables, actions, which can be used to manage the level of achieving benefits. These actions are assumed to have impacts on the perceptions of the user. Perceptions affect the attitude, positively or negatively, which lead to change in users' behaviour (Ajzen and Fishbein, 1977) (i.e., resistance to change or use). Behaviours and attitudes could be measured through indicators. Gap between targeted indicator level and the actual indicator level motivates BRP team to take corrective, balancing, actions in order to realign the actual adaptation process to the planned one. As a result, if system dynamics could indicate, through simulation, the targeted indicator level for attitude and behaviour to achieve a certain level in benefits, and if the BRP management should act in a way to balance the negative loops of consequences, system dynamics should be used to model BRP team actions to achieve the targeted level of benefits. Therefore, the aim of this research is to investigate how actions taken by BRP team affects the targeted level of benefits through managing organisational attitudes and perceptions toward the new ERP system using System Dynamics.

\section{LITERATURE REVIEW}

Benefits Management is "the process of organizing and managing such that the potential benefits arising from the use of IS/IT are actually realized" (Ward and Daniel, 2006). Based on this definition, the aim of benefit Realisation management is to achieve the targeted performance. Targeted performance is always planned according to the capacity and the ability of the information system only without taking into consideration the capacity and the ability of the people to change (Jenner, 2009).

People always resist to new changes. That is why they take time to adapt themselves to the new system. Adapting to new system is mainly based on the level of acceptance to the new system. Therefore, it is worth to mention earlier works in system acceptance and system success theories. On one hand, system acceptance theories focus on why, and how, do user, or organisation, accept or reject new system. On the other hand, system success theories focus on explaining the interdependence between different dimensions of success factors such as perceived ease of use, perceived usefulness, use, intention to use, and perceived net benefit. According to Delone \& McLean (2003), net benefits could be operationalized based on the context and the type of information system. Finally, representation theory (Burton-Jones and Grange, 2012) focuses on the adaptation process to achieve the effectiveness in the use.

On one side, current benefit Realisation models do not consider the adaptation rate factor. Adaptation rate is the speed of realizing benefits from the ERP system. Although system acceptance and success considers the social and psychological factors, these theories do not consider the actions required to achieve the targeted performance. Even though Burton-Jones \& Grange (2012) considers actions required to increase the adaptation rate, they focus only on learning variable. Therefore, Type and duration of learning, the way of managing the relationship with the vendor, and motivational ways for using the system are three factors that considered in this research to fulfil the research gap. Moreover, the research develops new benefit Realisation model that considers the human capacity required, i.e. psychological factors such as attitude; perception; and behaviour, for achieving targeted 
benefits. This research uses the relations in Table 1 to develop new benefit Realisation model based on organisational social capacity to adapt to the new ERP system.

Table 1: System Success and System Acceptance Theories

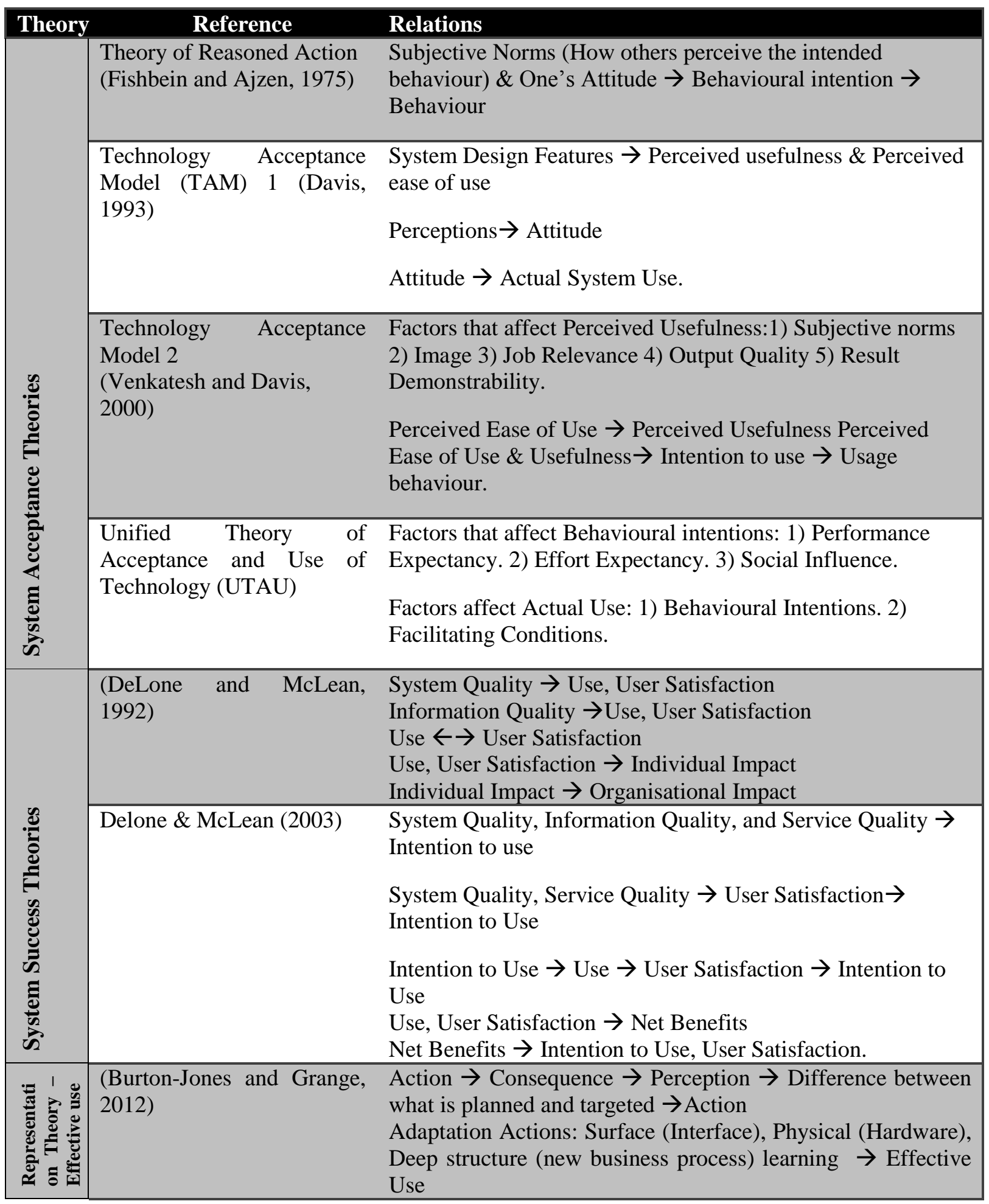

\section{SYSTEM DYNAMICS MODEL}

System Dynamics model is different from any other causal model since it takes into consideration a couple of important factors: namely, loops, either positive enforcing loops or negative balancing loops, and time lag, in terms of rates (Sterman, 2000). Since benefit management should be based on a loop of steps: identifying; planning; executing; and reviewing, this research is based on the same 
philosophy but with taken into consideration system acceptance and system success theories. Moreover, people could change at an adaptation rate to this new system and, therefore, to achieve the targeted benefits. Rate means that something over time factor. Therefore, system dynamics modelling is suitable for simulating and managing benefits targeted from ERP system.

Actually, behaviour is always driven by attitude. Additionally, attitude is based on what is perceived from experience, from background, or from learning process. Accordingly, affecting what is perceived could affect the attitude toward this object and this in turn leads to change in behaviour (Fishbein and Ajzen, 1975; Ajzen and Fishbein, 1977). What is perceived is affected by many factors such as information systems readiness, duration and content of learning process, the experience with using the system, and the relationship with the ERP vendor. These factors could be managed and controlled by BRP team in order to manage the behaviour of users toward the intended goal. Affecting attitude may be insufficient to manage the performance, or the effective use of the system, alone. Thus, deliberate motivational activities by BRP team should be used to increase the use. BRP team could use extrinsic motivations such as money and promotion or intrinsic motivations such as psychological rewards (e.g. recognition rewards, or certificates). Behaviour could take three forms: resistance to change, use, or effective use. Managing what is perceived should be directed to affect the behaviour to achieve the effective use behaviour.

The managing process of behaviour should take the closed- loop shape. As illustrated in Figure 1, this process should not only be based on actions, policies, that affect the adaptation level but also it should consider information regarding the current state in order to manage this process effectively.

To sum up, action, or policy, of BRP team should affect the perception of the organisation which affects the attitude level toward ERP. This attitude will be translated into behaviour. Positive attitude will lead to more use and effective use. On the other hand, negative attitude will lead to less use and more resistance to change. The general model could be illustrated in Figure 1.

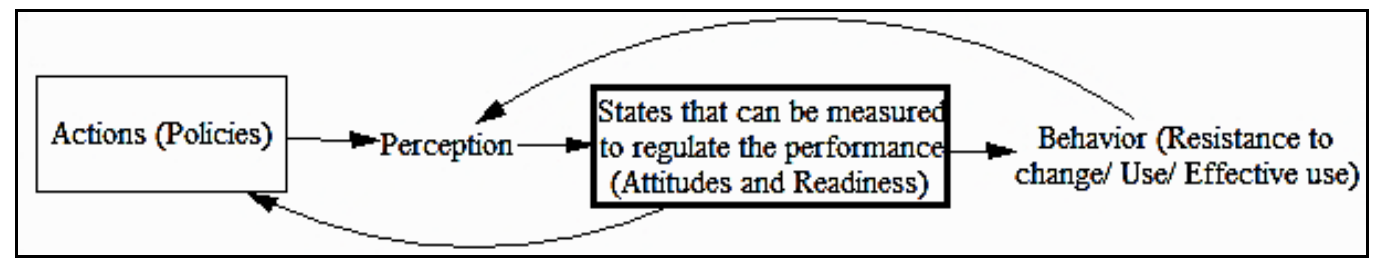

Figure 1: Benefit Realisation Management Model

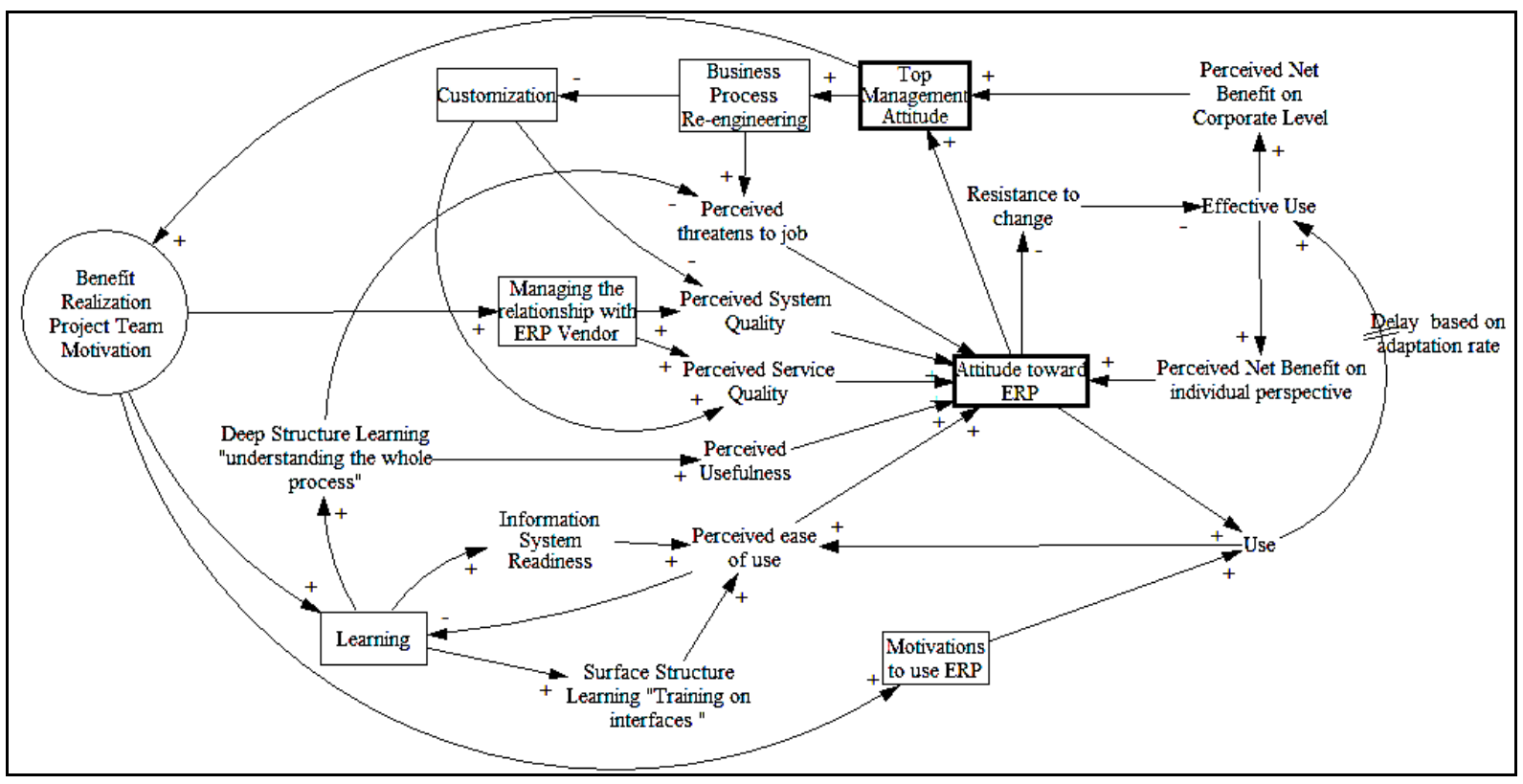

Figure 2: Benefit Realisation Management using System Dynamics Model 
The loop comes because this behaviour affect again on the perception. For example, more use will lead to more perceived ease of use. More perceived ease of use will affect positively on the organisational attitude toward the ERP system. Also the state level, attitude level, could be used as an indicator for BRP team to increase or decrease the level of actions such as learning duration, more close managing relationship with ERP vendors that could affect in turn the organisational perception toward ERP. According to Figure 2, Benefit Realisation Project Management Motivation circle is the level that affects all decisions and actions taken by BRP team. Black Boxes are the decisions, actions, or policies that taken by BRP in order to regulate and manage the state, the organisational attitude level. Black Bold Boxes are the attitude levels that should affect the behaviour. Other shapes are the behaviours and perceptions of the organisation toward different policies.

BRP Team has three controllable actions that could be used in order to increase the adaptation rate and; therefore, achieve the benefits effectively. Learning, managing the relationship with ERP vendor and motivations to use the system are three controllable policies. The fourth policy, the last resort for BPR team, is either business process re-engineering or customization the system since all efforts are failed to fit the organisation to ERP system. This decision is not easily taken since it requires top management acceptance.

Employees' learning process (Burton-Jones and Grange, 2012), either on ERP interface or on the understanding the new business processes, affects the perception of employees significantly. More hours training on interface, the more the perceived ease of the system will be. Additionally, not only perception of usefulness by employees will be from learning about the new business processes resulted from the use of ERP system, but also perceived threatens to their current jobs should be reduced because of the understanding how much they become useful and added value than before.

Managing relationship with vendor is very important for the perceived system quality and service quality. The more employees feel that their ERP related problems solved fast, the more the perception of service quality will be. In other words, the more the responsiveness, engaging, and commitment of ERP vendor, the more the service quality will be perceived by the organisation. Likewise, the more the actions lead to perceptions that system has less bugs or errors; the more the system quality is perceived by the organisation.

These perceptions are pooled cross time into the attitude pool. Attitude could be positive or negative toward the ERP System. Based on this attitude, users will use the system or users will resist to the new system. Although attitude is very an important factor in explaining the use behaviour, attitude may not be sufficient for motivating users to use the system. The policy of motivation should come into effect to motivate to use either in an intrinsic way such as certification for the best user or in an extrinsic way by linking the use behaviour with bonus and salaries.

Indeed, the use behaviour, in itself, affects the ease of use perception, since more use of the system; the more familiarity with it will be. The loop will until the use behaviour achieves a higher level which is the effective use. Effective use means using most of reports and features of the system which lead in turn to achieving the targeted benefits. Also an enforcing loop that affect the effective use behaviour which is positive attitude leads to reduction in resistance to change. This reduction in resistance will increase the effectiveness of the use. More effectiveness in the use will lead to perceived net benefits. This perception will lead to a positive attitude. This at the end will lead to a nested of virtuous loops.

The effectiveness in use by the organisation will lead to achievements of targeted organisational benefit from ERP. These achievements and the positive organisational attitude will be reflected on top management attitude toward the ERP system and the performance of BRP team. Certainly, the positive attitude will be reflected into more authority and more power to the BRP team. BRP team will be more involved and motivated to achieve more objectives.

\section{CONCLUSION}

Benefit Realisation process is a dynamics process; one action, or ignorance to take the action, may exacerbate the situation that leads to exponential speed toward success or failure in achieving the targeted benefits. The ability to manage organisational attitude and perception toward ERP system is a key factor for determining the rate of achieving targeted benefits. Perception of achieving targeted 
benefits becomes a feedback for closing the loop of BRP team motivation and managing the organisational attitude and perceptions through top management commitment toward the ERP system.

\section{ACKNOWLEDGMENT}

Egyptian Government and Cranfield University are thanked for funding this research project.

\section{REFERENCES}

Ajzen, I. and Fishbein, M. (1977), "Attitude-behaviour relations: A theoretical analysis and review of empirical research.", Psychological Bulletin; Psychological Bulletin, vol. 84, no. 5 , pp. 888 .

Bradley, G. (2010), Benefit Realisation Management: A Practical Guide to Achieving Benefits Through Change, Gower Publishing Company.

Breese, R. (2012), "Benefits realisation management: Panacea or false dawn?", International Journal of Project Management, vol. 30, no. 3, pp. 341-351.

Burton-Jones, A. and Grange, C. (2012), "From Use to Effective Use: A Representation Theory Perspective", Information Systems Research, vol. published online Nov 8, 2012.

Clegg, C., Axtell, C., Damodaran, L., Farbey, B., Hull, R., Lloyd-Jones, R., Nicholls, J., Sell, R. and Tomlinson, C. (1997), "Information technology: a study of performance and the role of human and organisational factors", Ergonomics, vol. 40, no. 9, pp. 851-871.

Coyle, R. G. (1996), System Dynamics Modelling: A Practical Approach, Chapman \& Hall, London.

Davenport, T. H. (1998), "Putting the enterprise into the enterprise system", Harvard Business Review, vol. 76, no. 4, pp. 121-131.

Davis, F. D. (1993), "User acceptance of information technology: system characteristics, user perceptions and behavioural impacts", International Journal of Man-Machine Studies, vol. 3, no. 38, pp. 475-487.

DeLone, W. H. and McLean, E. R. (1992), "Information systems success: The quest for the dependent variable", Information systems research, vol. 3, no. 1, pp. 60-95.

Fishbein, M. and Ajzen, I. (1975), Belief, attitude, intention and behaviour: An introduction to theory and research, Addison-Wesley, Reading, MA.

Jenner, S. (2009), "Realising Benefits from Government ICT Investment-a fool's errand", Reading, UK: Academic Publishing International Ltd, .

Nicolaou, A. I. (2004), "Firm performance effects in relation to the implementation and use of enterprise resource planning systems", Journal of Information Systems, vol. 18, no. 2, pp. 79-105.

Pellegrinelli, S., Partington, D. and Geraldi, J. (2011), "Programme management: An emerging opportunity for research and scholarship".

Shang, S. and Seddon, P. B. (2000), "A comprehensive framework for classifying the benefits of ERP systems", Americas Conference on Information Systems, pp. 1005.

Sterman, J. D. (2000), Business dynamics: systems thinking and modeling for a complex world, Irwin/McGraw-Hill, Boston.

Venkatesh, V. and Davis, F. D. (2000), "A theoretical extension of the technology acceptance model: Four longitudinal field studies", Management science, vol. 46, no.2, pp. 186-204.

Ward, J. and Daniel, E. (2006), Benefits Management: Delivering value from IS \& IT Investments, Wiley, England. 